Article

\title{
A Self-Referenced Diffraction-Based Optical Leaky Waveguide Biosensor Using Photofunctionalised Hydrogels
}

\author{
Anil K. Pal ${ }^{1}$, , Nicholas J. Goddard ${ }^{2, *}$, Hazel J. Dixon ${ }^{1}$ and Ruchi Gupta ${ }^{1, * \mathbb{D}}$ \\ 1 School of Chemistry, University of Birmingham, Birmingham B15 2TT, UK; a.k.pal@bham.ac.uk (A.K.P.); \\ hjd839@student.bham.ac.uk (H.J.D.) \\ 2 Process Instruments (UK) Ltd., Burnley BB12 0BT, UK \\ * Correspondence: nick.goddard@processinstruments.net (N.J.G.); r.gupta.3@bham.ac.uk (R.G.)
}

Received: 21 August 2020; Accepted: 21 September 2020; Published: 24 September 2020

check for updates

\begin{abstract}
We report a novel self-referenced diffraction-based leaky waveguide (LW) comprising a thin $(\sim 2 \mu \mathrm{m})$ film of a photofunctionalisable hydrogel created by covalent attachment of a biotinylated photocleavable linker to chitosan. Streptavidin attached to the chitosan via the photocleavable linker was selectively removed by shining $365 \mathrm{~nm}$ light through a photomask to create an array of strips with high and low loading of the protein, which served as sensor and reference regions respectively. The differential measurements between sensor and reference regions were used for measuring analytes (i.e., biotin protein $\mathrm{A}$ and $\mathrm{IgG}$ ) while reducing environmental and non-specific effects. These include changes in temperature and sample composition caused by non-adsorbing and adsorbing species, leading to reduction in effects by $\sim 98 \%, \sim 99 \%$, and $\sim 97 \%$ respectively compared to the absolute measurements. The novelty of this work lies in combining photofunctionalisable hydrogels with diffraction-based LWs for referencing. This is needed to realise the full potential of label-free optical biosensors to measure analyte concentrations in real samples that are complex mixtures, and to allow for sample analysis outside of laboratories where drifts and fluctuations in temperature are observed.
\end{abstract}

Keywords: self-referenced; leaky waveguide; biosensor; hydrogel; photocleavable

\section{Introduction}

Leaky waveguides (LWs) are a type of label-free optical biosensor, comprising of a few microns thick film with refractive index (RI) lower than the substrate but higher than the sample applied to the sensor [1-3]. LWs have been used for sensing different types of analytes including vapours [4], small molecules in solutions [5,6], microbes [7], and proteins [8-11]. In 2020, we observed a novel manifestation in LWs with low RI difference (0.002-0.005 RIU) between the waveguide and sample, and reported its suitability for the direct visualisation of the resonance angles as exponentially decaying interference fringes [12]. These LWs are called diffraction-based LWs [13] and offered 13 times higher figure of merit (FOM), which is the ratio of the refractive index sensitivity (RIS) and full width half maximum (FWHM) of the waveguide resonance, than surface plasmon resonance (SPR). Generally speaking, the higher the FOM, the easier it is to determine small shifts in the resonance angle observed at low analyte concentrations. Other benefits of LWs include ease of integration with electric fields for in-situ sample pre-concentration allowing for rapid analysis at low analyte concentrations [14], and fabrication using completely solution-processed methods and widely available materials for production of affordable biosensors. A limitation of the LWs is that their RIS is determined by the porosity of the waveguide layer to the analyte, and making mesoporous films with a thickness of a few microns is challenging [13]. 
The position of the resonance angle is a function of RI of the waveguide and the solution in its evanescent field. In addition to binding of analytes to recognition elements (REs) immobilised in LWs, the RI can also change because of common-mode effects i.e., variations in temperature, wavelength of light sources and sample composition. The temperature coefficient of RI of an aqueous solvent is $\sim 0.9 \times 10^{-4} \mathrm{RIU} /{ }^{\circ} \mathrm{C}$ at room temperature $[15,16]$, often resulting in much higher RI changes than that caused by analyte binding unless rigorous temperature control is applied. Temperature changes can also cause the peak wavelength of light sources to drift and "mode hops" in laser diodes [17]. Equally, the concentration of analytes is often several orders of magnitude lower than interferents in samples. The effect of changes in sample composition can be alleviated by integrating sample processing with biosensors for the removal of interferents and preconcentration of analytes. For example, electrokinetic sample processing has been integrated with LWs [14], but cannot compensate for changes in RI caused by other common-mode effects.

The contributions of analyte binding and common-mode effects can be separated by referencing [18-20] where typically differential measurements between the outputs of the sensor and reference regions are performed. Commercial instruments such as BIACORE have multiple fluidic channels where at least one of the channels is used for referencing [18]. The use of multiple fluidic channels often results in solutions reaching the sensor and reference channels at slightly different times, resulting in spikes in the differential signal. The presence of these spikes often makes it difficult to study the binding kinetics between the analyte and REs as well as to extract their association and dissociation constants. Sensor and reference regions have also been fabricated in a single fluidic channel by selective immobilisation of REs, which is typically achieved via microcontact printing [19-21]. Alternatively, electrolytic grafting of diazonium salts for selective immobilisation of REs in closed channel format has been reported for making referenced SPR biosensors [22]. The number of sensor and reference spots that can be fabricated using the approach is, however, determined by the number of available electrodes. In comparison to pre-fabricated electrodes, the use of light combined with digital photomasks is beneficial because the shape and distribution of sensor and reference regions can be rapidly changed on-demand. Towards this end, photocleavable linkers for patterning biomolecules on surfaces [23] and hydrogels [24] have been reported. The photolysis kinetics of these photocleavable linkers has been studied using label-free detection methods [23], but their application for the fabrication of sensor and reference regions of label-free optical biosensors has so far not been reported.

This is the first report of a diffraction-based LW with an array of alternate strips of different RI where the resonance angle corresponding to each strip was independently visualised as interference fringes. The ability to pattern low index hydrogels opens up the possibility of self-referencing and multiplexing while still retaining the high FOM of diffraction-based LW sensors. The strips were $\sim 1 \mathrm{~mm}$ wide and were fabricated by activating a chitosan waveguide with a photocleavable (PC) biotin linker, which was then allowed to bind to streptavidin. Subsequent photofunctionalisation by exposure to $365 \mathrm{~nm}$ light through a photomask (a Ronchi grating) selectively removed the linker and streptavidin. This work also demonstrates that the alternate strips with high and low loading of streptavidin can serve as sensor and reference regions. We showed that the differential response between these sensor and reference regions was suitable for analyte (in this work, $\operatorname{IgG}$ ) sensing while reducing the impact of undesirable side effects. The effect of changes in temperature over $5{ }^{\circ} \mathrm{C}$ and sample composition because of non-adsorbing and adsorbing species were reduced by $\sim 98 \%, \sim 99 \%$, and $\sim 97 \%$ respectively compared to the absolute shifts in the resonance angles. Our theoretical modelling also shows that diffraction-based LWs are significantly less dispersive [25] than other optical sensors (e.g., SPR [26] and resonant mirror (RM) [27]) and hence are less susceptible to variations in the wavelength of light sources. We can define a dispersion figure of merit (DFOM), which is the angular FWHM of the resonance peak or dip divided by the sensitivity of the resonance angle to wavelength, and has the units of wavelength. It is the wavelength change needed to move the resonance angle by the FWHM of the resonance. Table 1 gives the theoretical DFOM for SPR, RM and diffraction-based LWs operating at $650 \mathrm{~nm}$ obtained using transfer matrix modelling. It can be seen that the diffraction-based LWs, 
particularly when using TE polarised light, have the best DFOM, while the RM using TE polarised light provides the worst DFOM.

Table 1. Comparison of the theoretical dispersion characteristics of surface plasmon resonance (SPR), resonant mirror (RM) and diffraction-based leaky waveguides (LWs).

\begin{tabular}{cccc}
\hline Device & FWHM $\left(^{\circ}\right)$ & Sensitivity $\left({ }^{\circ} \mathbf{n m}^{-\mathbf{1}}\right)$ & DFOM (nm) \\
\hline SPR (TM) & 4.3185 & 0.0657 & 65.8 \\
RM (TM) & 1.0212 & $8.62 \times 10^{-3}$ & 118.5 \\
RM (TE) & 0.1941 & 0.0174 & 11.16 \\
Diffraction-based LW (TM) & 0.0518 & $3.62 \times 10^{-4}$ & 143.1 \\
Diffraction-based LW (TE) & 0.0476 & $1.60 \times 10^{-4}$ & 296.7 \\
\hline
\end{tabular}

\section{Experimental}

\subsection{Materials}

$1 \mathrm{M}$ acetic acid, $1 \mathrm{M}$ sodium hydroxide, methanol, acetone, 25\% (v/v) glutaraldehyde (GA), glycerol $\left(\mathrm{M}_{\mathrm{w}}\right.$ : 92), poly(ethylene glycol) (PEG) $\left(\mathrm{M}_{\mathrm{w}}: 10,100\right.$ and $\left.300 \mathrm{kD}\right)$, protein A-biotin (PAB) from Staphylococcus aureus (P2165), IgG from sheep serum (I5131), semicarbazide hydrochloride (ScZ), sodium phosphate monobasic monohydrate, sodium phosphate dibasic dodecahydrate, bovine serum albumin (BSA), and anhydrous dimethyl sulfoxide (DMSO) were purchased from Sigma Aldrich (UK). HEPES and decon 90 were purchased from Fisher Scientific (UK). Streptavidin (2-0203-100) was purchased from IBA Lifesciences (Germany). PC Biotin-PEG $3-\mathrm{NHS}$ carbonate ester (PC-biotin) with purity of $\sim 95 \%$ was purchased from BroadPharm (USA). The above-purchased chemicals were used as received without further purification. Chitosan $\left(\mathrm{M}_{\mathrm{w}}\right.$ : 100-300 kD, 90\% deacetylated) was purchased from Sigma Aldrich (UK) and purified by following the steps described in our previous report [28]. Glass microscope slides were purchased from VWR (UK), dialysis tubing cellulose membrane (molecular weight cut-off: 14,000) was purchased from Sigma Aldrich (UK), and syringe filters with cut-off size of $5 \mu \mathrm{m}$ were purchased from Scientific Lab Suppliers (UK). $100 \mathrm{mM}$ phosphate buffer (PB) solution at $\mathrm{pH} 8.5$ was prepared by dissolving appropriate amounts of sodium phosphate monobasic and dibasic salts in de-ionised (DI) water. $100 \mathrm{mM}$ HEPES buffer solution of pH 7.4 was also prepared.

\subsection{Fabrication of Diffraction-Based LWs and Their Photopatterning}

The glass substrates of $\sim 25 \times 25 \mathrm{~mm}^{2}$ were cleaned in decon 90 solution, DI water, and ethanol by sonication for $30 \mathrm{~min}$ each, and then dried in a hot air oven operated at $60{ }^{\circ} \mathrm{C}$. Finally, the substrates were cleaned using a plasma cleaner (Harrick Plasma, PDC-002-CE).

The purified chitosan was dissolved in $0.1 \mathrm{M}$ acetic acid under continuous stirring for $18 \mathrm{~h}$ to form a $1 \%(w / v)$ solution [28]. $150 \mu \mathrm{L}$ of chitosan solution was spin coated onto the glass substrate at a spin speed of $900 \mathrm{rpm}$ for $30 \mathrm{~s}$ with acceleration of $100 \mathrm{rpm} / \mathrm{s}$ inside a laminar flow cabinet. The spin coated substrates were placed inside an incubator maintained at $25^{\circ} \mathrm{C}$ and humidity of $75-80 \%$ for $3 \mathrm{~min}$. The chitosan films were then crosslinked by immersing them in $0.03 \%(v / v)$ GA prepared in HEPES buffer for $5 \mathrm{~min}$, washed with HEPES buffer to remove unreacted GA, and stored in HEPES buffer until further use.

Chitosan films were treated with $500 \mu \mathrm{L}$ of PC-biotin of different concentrations for $40 \mathrm{~min}$ and then washed with HEPES buffer. Subsequently, the films were treated with streptavidin $(0.05 \mathrm{mg} / \mathrm{mL})$ for 40 min and washed with HEPES buffer. Finally, the chitosan films with immobilised streptavidin were immersed in 1.25\% (w/v) ScZ prepared in HEPES buffer. The light emitted by a $365 \mathrm{~nm}$ LED (Nichia NVSU233A-U365, $1030 \mathrm{~mW}$ ) was collimated by passing through a $25 \mathrm{~mm}$ diameter planoconvex lens, resulting in a beam with a power density of $\sim 210 \mathrm{~mW} \mathrm{~cm}^{-2}$. The collimated beam was passed through a Ronchi grating of pitch $1 \mathrm{~mm}$, and the resulting pattern of bright and dark lines was projected perpendicularly on the chitosan films for different durations. 


\subsection{Preparation of Solutions for Characterisation of LWs}

Glycerol and different PEG $\left(\mathrm{M}_{\mathrm{w}}: 10,100\right.$ and $\left.300 \mathrm{kD}\right)$ solutions were prepared in HEPES buffer and used to estimate the porosity of chitosan LWs. The received lyophilised powder of PC-biotin was dissolved in anhydrous DMSO in an inert atmosphere $\left(\mathrm{N}_{2}\right.$ gas) at $10 \mathrm{mg} / \mathrm{mL}$ and stored in a freezer in darkness for further use. The aliquots were diluted in HEPES buffer before use to obtain PC-Biotin solutions of different concentrations. BSA, streptavidin, PAB, and IgG solutions were prepared in HEPES buffer with concentrations of $1,0.05,0.07$, and $0.1 \mathrm{mg} / \mathrm{mL}$, respectively.

\subsection{Instrumentation}

As shown in Figure 1, light from a point source red LED (TL-6, iC-Haus, $640 \mathrm{~nm}$ ) was passed through optical lenses ( $40 \mathrm{~mm}$ focal length achromatic doublet and $63 \mathrm{~mm}$ focal length cylindrical lens) to illuminate the LW with a wedge-shaped light beam. The LW was mounted on a BK7 equilateral prism (Qioptic Photonics, Denbighshire, UK) using a RI matching oil and the reflected light was captured using a 20 Mpixel CMOS camera (MER-2000-19U3M-L, Daheng Imaging, Beijing, China). A single channel flow cell was made from $3 \mathrm{~mm}$ thick poly(methyl methacrylate) (PMMA) and the dimensions of the channel are provided in the inset in Figure 1. The flow cell was mounted on the top of the LW and held in place using a clamping plate connected to a temperature-controlled recirculating water bath. The solutions used for testing the chitosan LWs were pumped through the flow cell using a peristaltic pump (Minipuls ${ }^{\circledR}$ 3, Gilson, Bedfordshire, UK) at a flow rate of $200 \mu \mathrm{L} / \mathrm{min}$. The RI of the solutions were measured using RFM900-T refractometer (Bellingham and Stanley, Kent, UK) with an accuracy of $\pm 1 \times 10^{-5}$ RIU.

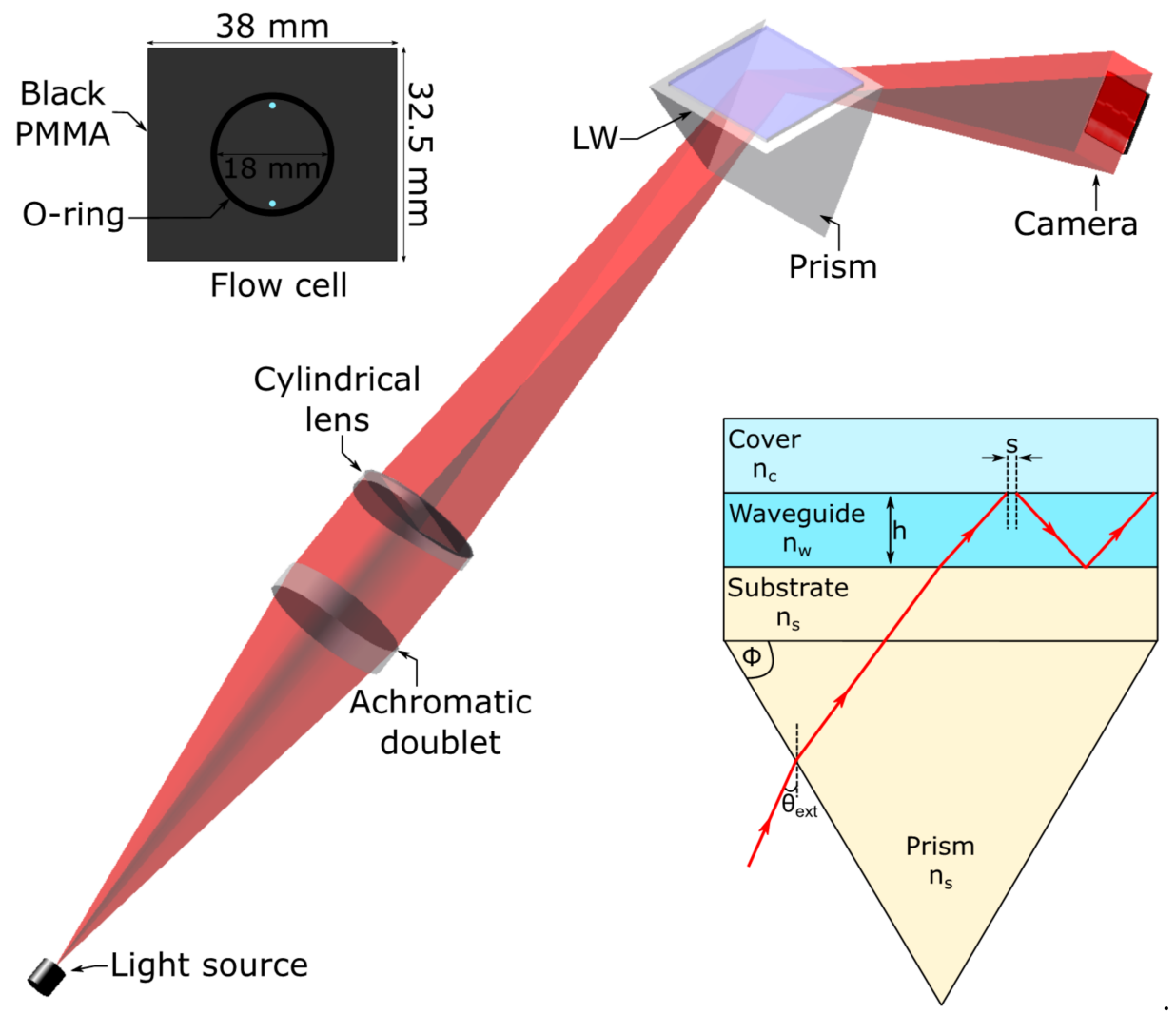

Figure 1. Schematic of the LW instrumentation (the base of the prism is $30 \mathrm{~mm}$ by $30 \mathrm{~mm}$ ) where the insets show the flow cell used in this work, and a ray diagram showing partial confinement of light in LWs (where $\theta_{\text {ext }}$ is the external angle of incidence, $s$ is the Goos-Hänchen shift, and $n_{c}, n_{w}, n_{s}$ are the refractive indices of cover/sample, waveguide and substrate respectively. For LWs, $\mathrm{n}_{\mathrm{c}}<\mathrm{n}_{\mathrm{W}}<\mathrm{n}_{\mathrm{s}}$ ). 


\section{Results and Discussion}

\subsection{Optimisation of the Diffraction-Based LW}

A typical output of a diffraction-based LW with an unpatterned chitosan film is shown in Figure 2a where interference fringes were observed at the resonance angle $\left(\theta_{R}\right)$. The $\theta_{R}$ was $\sim 1.211^{\circ}$ away from the total internal reflection (TIR) angle. The corresponding reflectivity curve is provided in Figure $2 \mathrm{~b}$ and the FWHM of the dip-peak pair was $\sim 0.192^{\circ}$. As discussed in our previous work [12], for LWs where the RI between the waveguide and sample solution is $<0.01$, exponentially decaying interference fringes are observed at resonance angles. The reflectivity curve was normalised to the intensity between 63.5 and $65^{\circ}$ because the reflectivity in this region is expected to be unity. The reflectivity of the interference peak is greater than one because of constructive interference, while the reflectivity of the interference dip is less than one because of destructive interference. At angles greater than the resonance angles, the reflectivity is one because of TIR. The small hump in the grey-scale values observed between $\sim 66$ and $67^{\circ}$ in Figure $2 b$ is a result of the stray reflections that were observed as a white streak in Figure 2a.
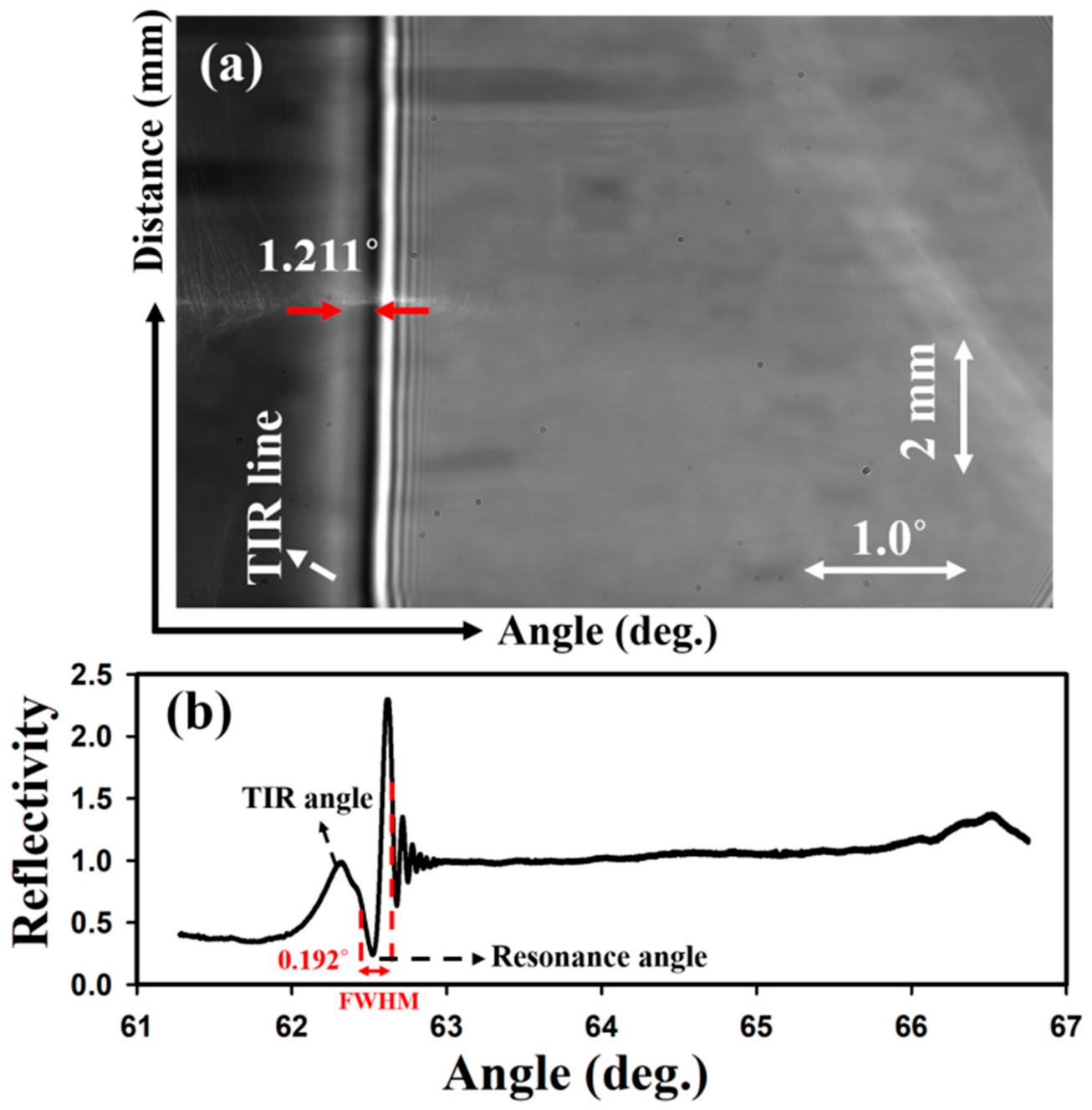

Figure 2. (a) Output of a chitosan LW with flow cell mounted on the top and filled with HEPES buffer, and (b) the corresponding reflectivity curve (where angle is $\theta_{\text {ext }}$ as marked in Figure 1).

A simplex optimisation was performed to extract the thickness and RI of the chitosan LWs from the experimental reflectivity curves. The typical thickness and RI of the chitosan films were estimated to be $1.95 \mu \mathrm{m}$ and $1.3437-1.28 \times 10^{-4} \mathrm{i}$ respectively. The imaginary RI of the chitosan films indicates 
that there may be some scattering losses. The RIS of the chitosan LW was $121.09 \pm 4.30^{\circ} \mathrm{RIU}^{-1}$ (see Figure S1 in Supplementary Materials for details). The FOM was $630.68 \pm 22.40 \mathrm{RIU}^{-1}$, which is $\sim 1.8$ times lower than poly(acrylamide) LWs [12], and may be because of higher scattering losses in the chitosan than poly(acrylamide) films. In all cases, the error bars represent area-to-area variability across the width of the flow cell mounted on the LW. This area-to-area variability across the sensor surface arose from the variations in the waveguide thickness resulting from the spin coating process. Within an experimental run using a single device, the primary factor determining the measurement noise is the pressure pulsation introduced by the peristaltic pump used to drive solutions into the flow cell. The device-to-device reproducibility is primarily determined by the waveguide material, which, in this case, is a natural material (chitosan) and has inherent variability that is difficult to quantify.

Figure S2 in Supplementary Materials shows the response for glycerol (92 D) and PEG (10, 100 and $300 \mathrm{kD}$ ) solutions of $10 \mathrm{mg} / \mathrm{mL}$. It is observed that the shift in the resonance angle for glycerol is $0.16 \pm 0.01^{\circ}$ while for 10,100 and $300 \mathrm{kD} \mathrm{PEG}$ solutions is $0.14 \pm 0.01^{\circ}, 0.134 \pm 0.01^{\circ}$, and $0.13 \pm 0.01^{\circ}$, respectively. The RI values of each solution are also provided in Figures S1 and S2 in Supplementary Materials. The RIS for each solution was estimated by taking a ratio of the shift in resonance angle and the RI of the solution. Subsequently, the porosity was estimated using Equation (1) [13]:

$$
P=\frac{R I S_{p}-R I S_{e w}}{R I S_{g l y}-R I S_{e w}} \times 100 \%
$$

where $R I S_{p}$ is the refractive index sensitivity for a given PEG solution, $R I S_{e w}$ is the sensitivity to refractive index in the evanescent field and $R I S_{g l y}$ is the sensitivity to glycerol. $R I S_{e w}$ is determined by modelling using the thickness and refractive index of the waveguide. Based on diffusion studies (see Figure S2 in Supplementary Materials for details) and Equation (1), the chitosan films had a porosity relative to glycerol of $93 \%, 81 \%$ and $76 \%$ for $10 \mathrm{mg} / \mathrm{mL}$ solutions of PEGs of molecular weight 10,100 and $300 \mathrm{kD}$.

Next, we optimised the concentration of the PC-biotin solution used to treat the chitosan films. Figure 3 shows that as the concentration of PC-biotin solution was increased, larger amounts of streptavidin were immobilised in the waveguide, which was reflected as a bigger shift of the resonance angle $\left(\Delta \theta_{R}\right)$ of the LWs. The angle shifts of $0.44 \pm 0.01^{\circ}, 0.75 \pm 0.02^{\circ}$ and $1.93 \pm 0.02^{\circ}$ for $0.47,1$, and $2 \mathrm{mg} / \mathrm{mL}$ PC-biotin respectively correspond to streptavidin concentrations $\left(c_{\text {strep }}\right)$ of $0.35 \pm 0.01$, $0.59 \pm 0.02$ and $1.53 \pm 0.02 \mathrm{mM}$ respectively based on the RIS of the LW sensor, a RI increment $(\delta n / \delta c)$ of $0.19 \mathrm{~g}^{-1} \mathrm{~mL}$ of typical proteins [29] and using Equation (2) where $M_{\text {strep }}$ is molecular weight of streptavidin.

$$
c_{\text {strep }}=\frac{\Delta \theta_{R}}{M_{\text {strep }} \times R I S \times(\delta n / \delta c)}
$$

The concentration of amines $\left(c_{\text {amines }}\right)$ in chitosan is given by Equation (3).

$$
c_{\text {amines }}=\frac{\mathcal{c}_{\text {chitosan }}}{D \times M_{g}+(1-D) \times M_{a g}}
$$

where $c_{\text {chitosan }}$ is $10 \mathrm{~g} / \mathrm{L}$ i.e., concentration of chitosan solution used to make the films, $D$ is degree of deacetylation (90\%), $M_{g}$ is the molecular mass of a glucosamine unit and $M_{a g}$ is the molecular mass of an acetylglucosamine unit. The concentration of amines in the film was estimated to be $\sim 60 \mathrm{mM}$. The ratio of $c_{\text {strep }}$ and $c_{\text {amines }}$ was then calculated, and only a maximum of $2.5 \%$ of the available amines in chitosan were occupied by streptavidin. However, as the concentration of PC-biotin solution was increased to $2 \mathrm{mg} / \mathrm{mL}$, a broad and shallow dip in reflectivity was observed at the resonance angle of the LWs after streptavidin binding (see Figure $3 \mathrm{c}, \mathrm{f}$ ). This can be explained by considering that the streptavidin binding increased the RI of the chitosan waveguide. As the RI of the waveguide increases, the fraction of light that leaks at the waveguide/substrate interface increases, which means that the optical losses in the waveguide increase. These increased losses cause the dip-peak pair at 
the resonance angle to be replaced by a broad shallow dip, which is quite hard to track to monitor changes in the resonance angle for sensing. This shallow dip is consistent with our previous work [12], which showed that increasing the RI of the waveguide resulted in a decrease in the amplitude of the interference fringes, which eventually disappeared when the RI contrast between the waveguide and sample exceeded 0.01 .
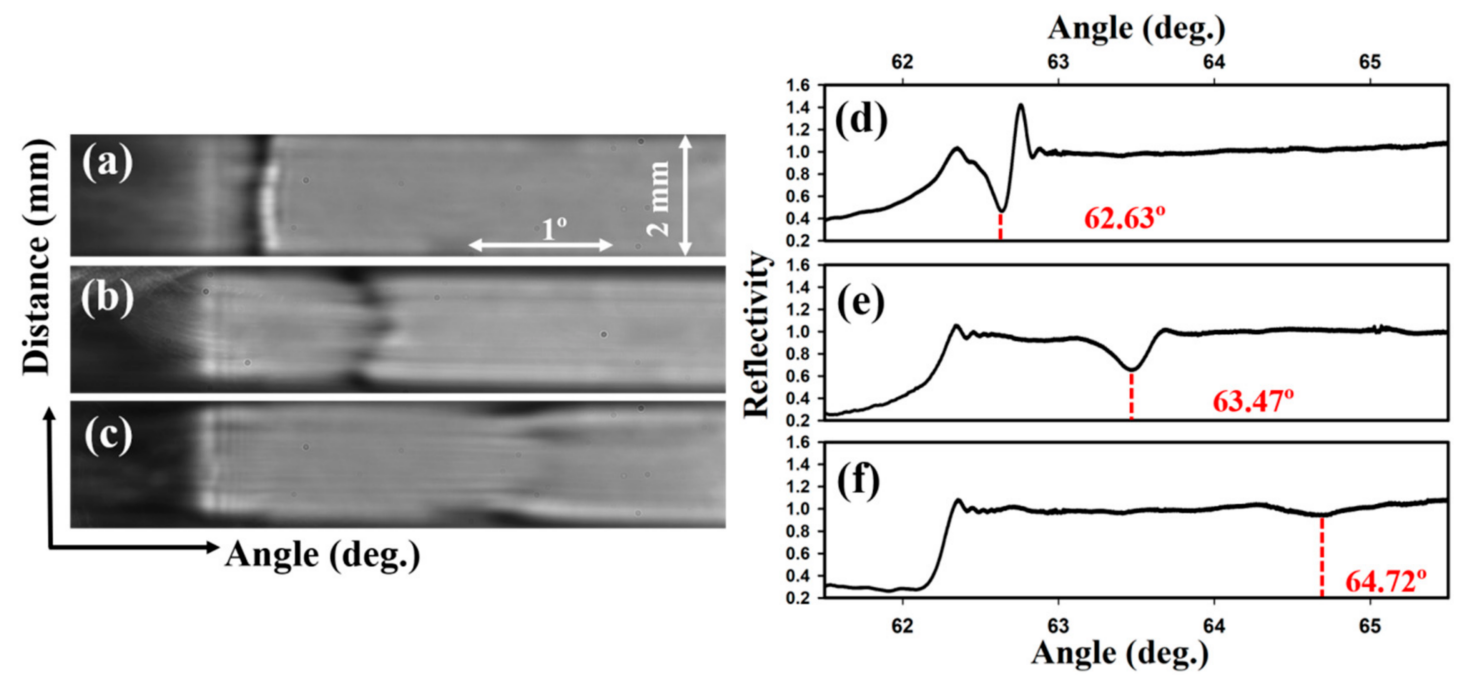

Figure 3. 2D and the corresponding 1D reflectivity of LWs after streptavidin immobilisation to chitosan films activated with (a,d) $0 \mathrm{mg} / \mathrm{mL},(\mathbf{b}, \mathbf{e}) 1 \mathrm{mg} / \mathrm{mL}$ and $(\mathbf{c}, \mathbf{f}) 2 \mathrm{mg} / \mathrm{mL}$ of PC-biotin (where angle is $\theta_{\text {ext }}$ as marked in Figure 1).

To create active and inactive regions for analyte binding in the $\mathrm{LW}$, a photocleavable biotin reagent, $1 \mathrm{mg} / \mathrm{mL}$ PC-biotin solution was used. It comprises a PEG spacer arm with a nitrobenzyl photocleavable moiety linked to an amine reactive N-hydroxysuccinimidyl (NHS) group at one end, and a biotin moiety at the other end. When the chitosan film was treated with PC-biotin, the NHS region reacted specifically with the primary amines $\left(-\mathrm{NH}_{2}\right)$ of chitosan. Streptavidin was then bound to the biotin end of the photocleavable linker. Illumination with near-UV light cleaved the linker, removing the streptavidin from the exposed area, allowing selective immobilisation of biotinylated species (i.e., protein A-biotin, PAB) in the next step.

Figure 4 shows the output image of a chitosan LW (Figure 4a), the LW after immobilisation of streptavidin (Figure 4b), and the photopatterned LW (Figure 4c-e), with alternating active (presence of streptavidin) and inactive (absence of streptavidin) regions for exposure times of 5, 8, and $15 \mathrm{~min}$, respectively. $\Delta \theta_{R}(0)$ and $\Delta \theta_{R}(t)$ in Figure $4 \mathrm{f}, \mathrm{g}$ are defined in Equations (4) and (5), respectively.

$$
\begin{gathered}
\Delta \theta_{R}(0)=\theta_{\text {strep }}-\theta_{w g} \\
\text { And } \Delta \theta_{R}(t)=\theta_{t}-\theta_{w g}
\end{gathered}
$$

where $\theta_{w g}$ is the resonance angle before streptavidin immobilisation (shown in Figure $4 \mathrm{a}$ ), $\theta_{\text {strep }}$ is the resonance angle after streptavidin immobilisation (shown in Figure $4 \mathrm{~b}$ ) and $\theta_{t}$ is the resonance angle after exposure to $365 \mathrm{~nm}$ light for time ' $\mathrm{t}$ ' (shown in Figure 4c-e). 

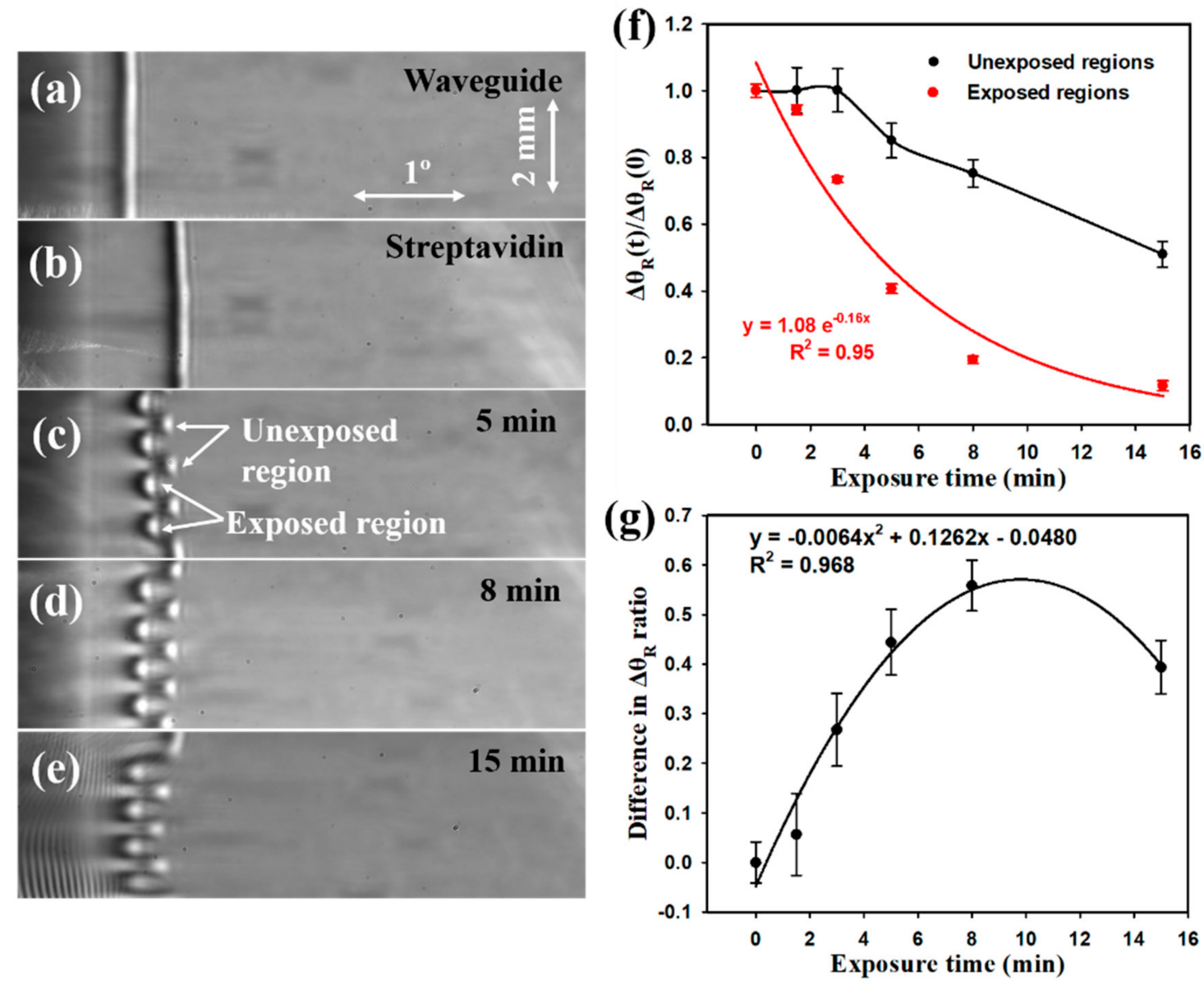

Figure 4. Output of a diffraction-based LW (a) before and (b) after streptavidin immobilisation, and its selective removal by exposure to $365 \mathrm{~nm}$ light for (c) $5 \mathrm{~min}$, (d) $8 \mathrm{~min}$ and (e) $15 \mathrm{~min}$, (f) ratio and (g) absolute difference of $\Delta \theta_{\mathrm{R}}$ after and before photo exposure versus exposure time.

As shown in Figure $4 \mathrm{f}$, the $\Delta \theta_{R}(t) / \Delta \theta_{R}(0)$ for the exposed regions decreased exponentially as the exposure time was increased. The unexposed region was expected to show a slower exponential decay, but the relatively large error bars make it difficult to determine the relationship between $\Delta \theta_{R}$ and exposure time. The exposure time of $1.5 \mathrm{~min}$ released $\sim 6 \%$ of streptavidin and a maximum of $\sim 88 \%$ was released for exposure time of $15 \mathrm{~min}$. Although the exposure time of $15 \mathrm{~min}$ removed a significant fraction of streptavidin $(\sim 88 \%)$ from the exposed regions of the waveguide, it also removed up to $\sim 49 \%$ of streptavidin from the unexposed area, leaving fewer active binding sites for the sensing of protein A-biotin (PAB) and IgG. A plot of the difference in the ratios of $\Delta \theta_{\mathrm{R}}$ from unexposed regions to exposed regions against exposure time (see Figure $4 \mathrm{~g}$ ) followed a parabolic profile with a peak at the exposure time of $9.9 \mathrm{~min}$, which is close to the experimental value of $8 \mathrm{~min}$. For this reason, $8 \mathrm{~min}$ exposure was used in the subsequent work.

There is no upper limit on the grating period, but the lower bound on the grating period was limited by the ability to visualise the resonance angle corresponding to the individual strips. As can be seen in Figure $4 c$,d, the higher RI of the unexposed strips causes additional diffraction at the strip edges, which means that the resonance angle of the individual strips become harder to resolve as the strip width decreases. A grating period of $2 \mathrm{~mm}$ was found to be optimum.

\subsection{Self-Referenced Diffraction-Based LW Biosensor}

The reflectivity profiles of a diffraction-based LW for regions unexposed and exposed (i.e., with high and low streptavidin loading, respectively) to $365 \mathrm{~nm}$ light are shown in Figure 5. Figure 5 
clearly shows that the resonance angles corresponding to the LW with high and low streptavidin loading are different. The feasibility of a diffraction-based LW with alternating regions of high and low streptavidin loading for referencing was investigated next. To do this, absolute and differential shifts in the resonance angles of high and low streptavidin loading (i.e., sensor and reference) regions to common-mode effects (i.e., temperature, sample composition caused by non-adsorbing and adsorbing species) and analyte binding were determined, and the results are discussed below.

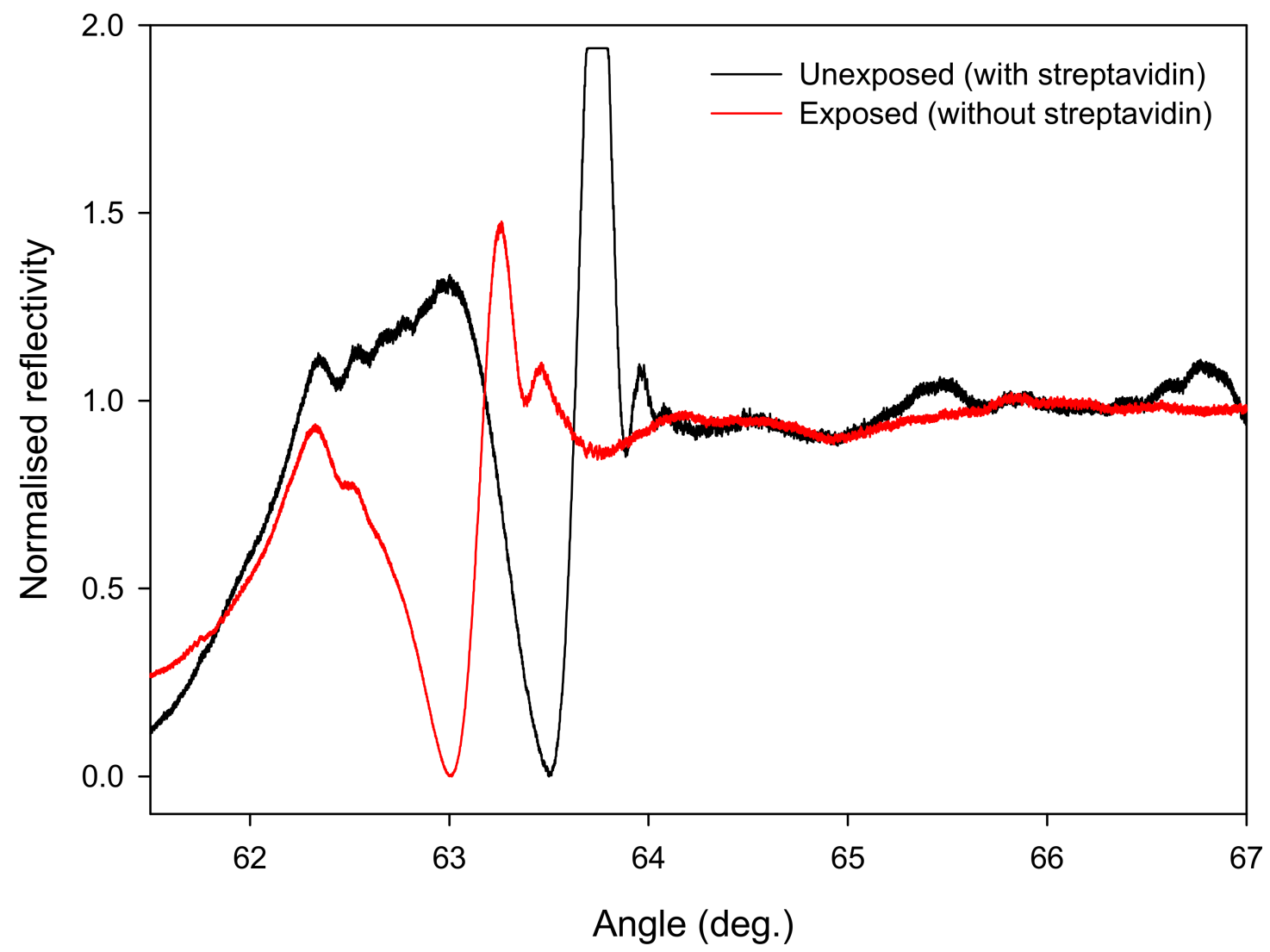

Figure 5. Normalised reflectivity curves of the regions of diffraction-based LWs with and without immobilised streptavidin (where angle is $\theta_{\text {ext }}$ as marked in Figure 1).

\subsubsection{Compensation for Changes in Temperature}

The temperature of the water bath used to recirculate the fluid through the plate mounted on top of the flow cell and LW assembly was increased from $20^{\circ} \mathrm{C}$ to $25^{\circ} \mathrm{C}$ in steps of $2.5^{\circ} \mathrm{C}$. As shown in Figure $6 \mathrm{a}$, the absolute shifts in resonance angles of sensor and reference regions decrease by $\sim 0.08^{\circ}$ as the temperature increases from $20^{\circ} \mathrm{C}$ to $25^{\circ} \mathrm{C}$. Figure $6 \mathrm{a}$ also shows that the shifts in the resonance angles of the sensor and reference regions because of temperature changes were approximately equal. The differential signal produced by this temperature change was $\sim 98 \%$ lower than the absolute shifts in the resonance angles of the sensor and reference regions. Thus, differential measurements between the shifts in the resonance angles of the sensor and reference regions can greatly reduce the temperature effect from the overall LW output. The unexposed regions seem to respond slightly less rapidly to changes in temperature than the exposed regions. It seems unlikely that this is because of differences in the thermal diffusivity or heat capacity of the very thin chitosan layer, so might be a result of physical changes in the hydrogel because of the difference in water content. 
Time (s)
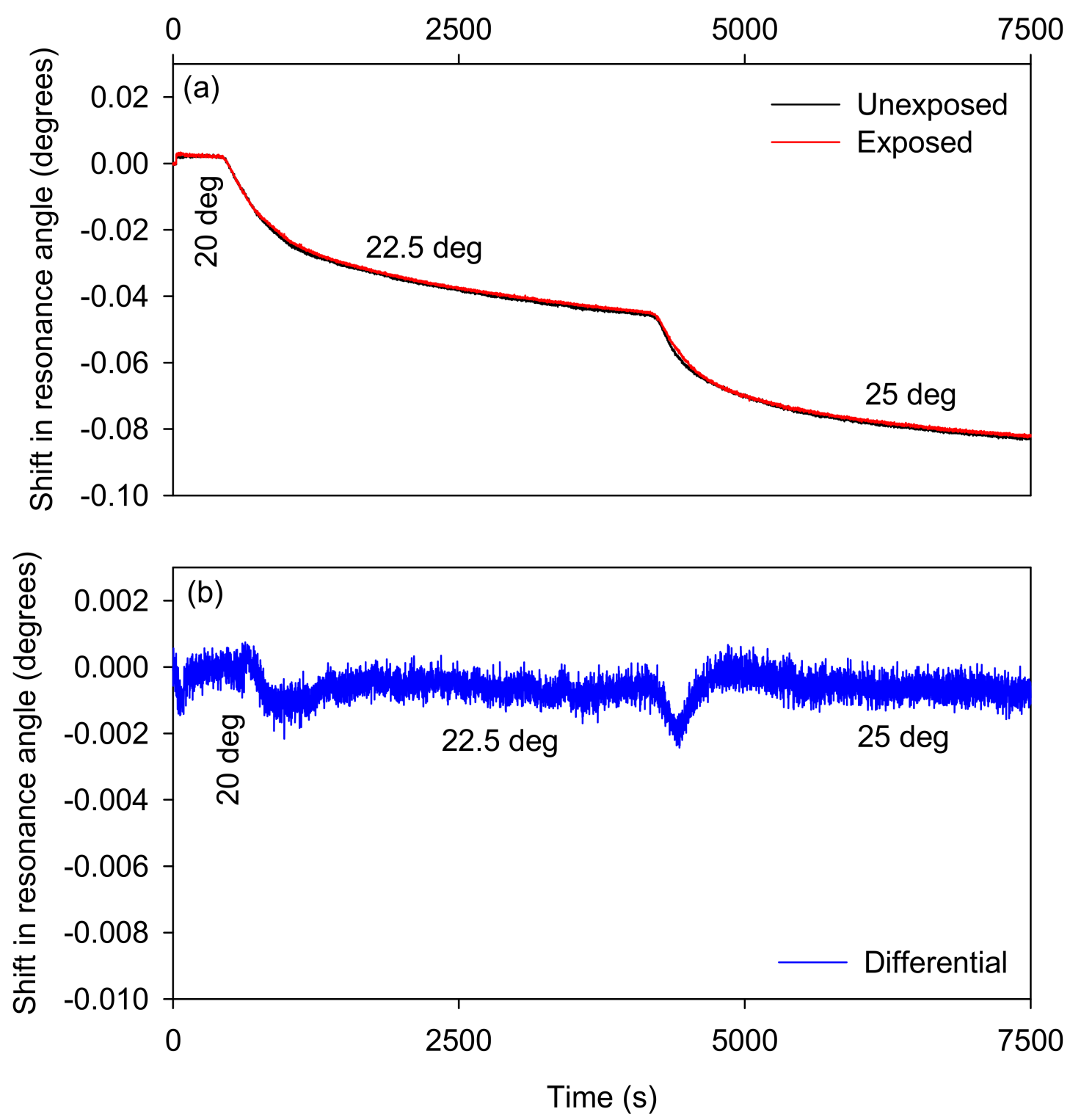

Figure 6. (a) Absolute and (b) differential shifts in the resonance angles of the self-referenced diffraction-based LW when the system temperature was varied.

\subsubsection{Compensation for Changes in Sample Composition}

- Non-adsorbing interferents: Figure 7 shows the absolute and differential shifts in resonance angles as a result of changes in RI caused by different concentrations of glycerol solutions. The RI of glycerol solutions of different concentrations are provided in Table S1 in Supplementary Materials. On average, the differential response because of changes in bulk RI was $~ 99 \%$ lower than the absolute shifts in the resonance angles of the sensor and reference regions. 
Time (s)
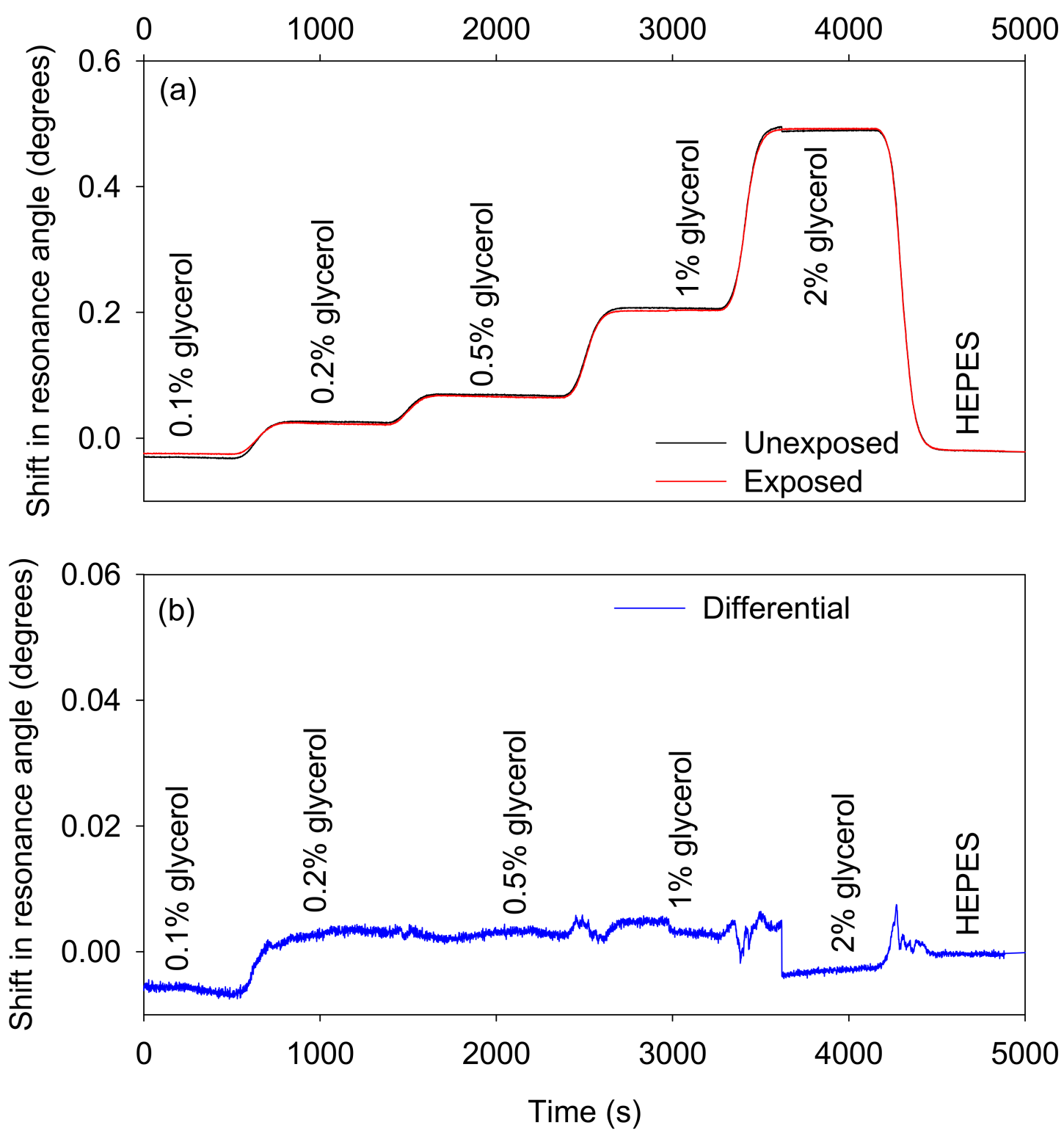

Figure 7. (a) Absolute and (b) differential shifts in the resonance angles of the self-referenced diffraction-based LW to changes in sample composition caused by different concentrations of glycerol solutions.

- Adsorbing interferents: Ideally, species in samples should not non-specifically adsorb to the waveguide materials. Alternatively, the non-specific adsorption of species to sensor and reference regions should at least be similar so that differential measurements can be performed to remove the contribution of non-specific adsorption from the overall output of the biosensor. We investigated the potential of the reported self-referenced LW to eliminate the effect of non-specific adsorption by using $1 \mathrm{mg} / \mathrm{mL}$ BSA solution prepared in $100 \mathrm{mM}$ HEPES buffer, pH 7.4. As shown in Figure 8a, the resonance angle of sensor and reference regions increased as BSA solution was introduced on top of the patterned chitosan LW. The resonance angles did not return back to baseline after a buffer wash. This may be a result of electrostatic interactions between BSA and the chitosan films as well as hydrophobic interactions between BSA and the NVOC in unexposed regions of the film. The isoelectric point of BSA is 4.7-5.1 [30], whereas the $\mathrm{pK}_{\mathrm{a}}$ of the amines in chitosan is 
about 6.5 [31], which means that BSA will be negatively charged at the selected $\mathrm{pH}$ of the buffer, and hence can interact with positively charged primary amines in chitosan films [32]. Since the difference in response to BSA between the exposed and unexposed regions is quite small, it seems likely that electrostatic interactions are more significant. The shifts in resonance angles of sensor and reference regions because of non-specific adsorption of BSA were, however, similar. As a result, as shown in Figure 8b, the differential signal was up to $97 \%$ lower than the absolute shifts in the resonance angles of the sensor and reference regions. The remaining small difference between the exposed and unexposed regions can be explained by non-specific interaction of BSA with the higher concentration of streptavidin in the unexposed regions.

Time (s)
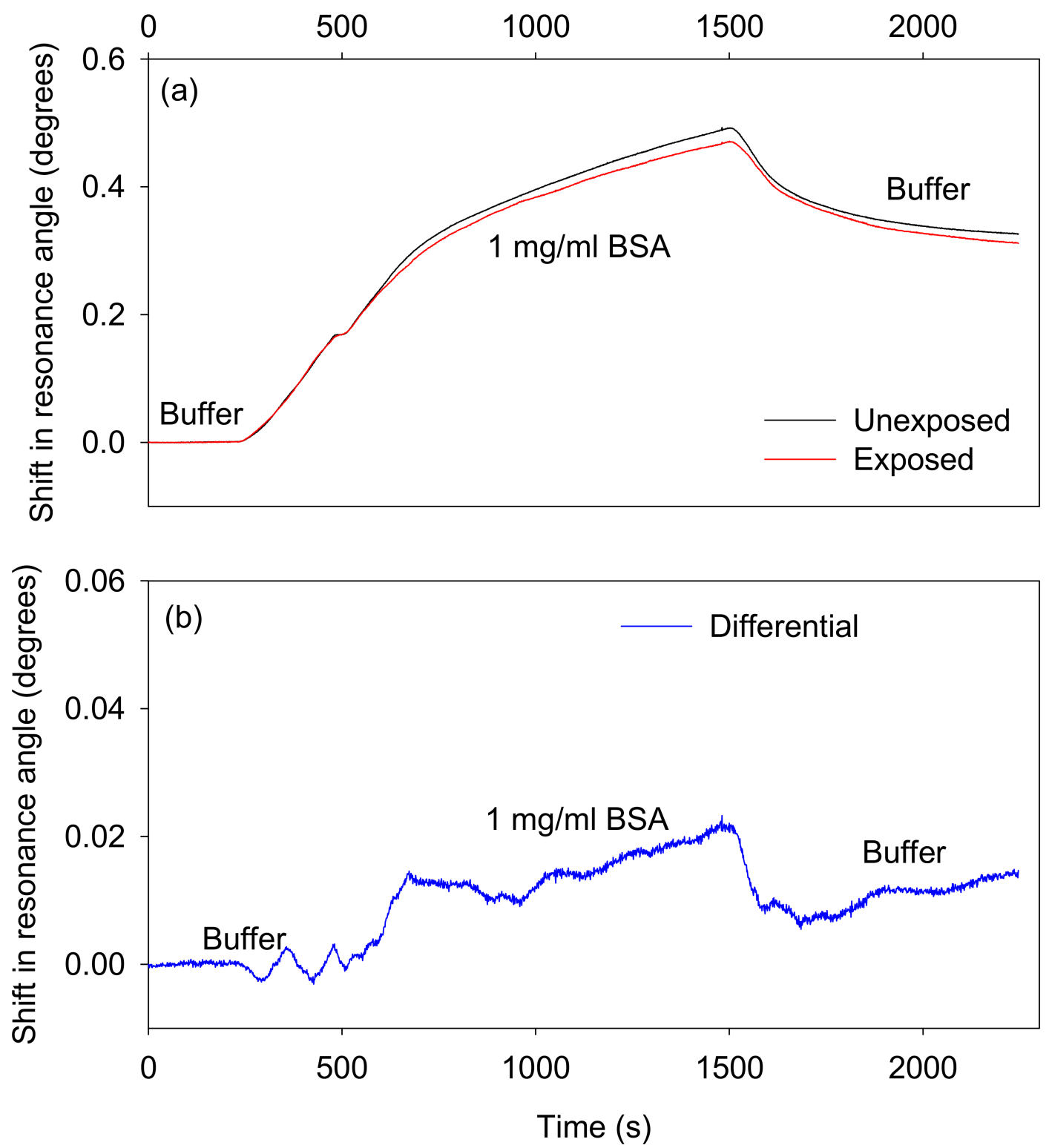

Figure 8. (a) Absolute and (b) differential shifts in the resonance angles of the self-referenced diffraction-based LW to non-specific adsorption of $1 \mathrm{mg} / \mathrm{mL}$ BSA prepared in $100 \mathrm{mM}$ HEPES buffer, $\mathrm{pH} 7.4$. 


\subsubsection{Kinetic Analysis of Analyte Binding}

The difference in the concentration of streptavidin in the exposed and unexposed regions will make some difference to the kinetics of analyte binding, for two reasons. Firstly, removal of streptavidin will make a difference to the porosity of the film, changing the rate at which other molecules can diffuse into the film and bind to the remaining streptavidin. Secondly, we can assume a laminar flow regime with a Nernstian diffusion layer, based on the calculated Reynolds number of 0.74 for the flow cell and flow velocity used. In this case, the surface concentration of binding sites will make a difference to the rate at which analyte can bind. The expression for the time dependence of the surface concentration of bound analyte is given by Equation (6) [33], where $D$ is the analyte diffusion coefficient, $K$ the dissociation constant for the binding pair, $t$ is time, $\delta$ is the thickness of the diffusion layer and $\Gamma_{\text {lim }}$ the surface concentration of streptavidin.

$$
\frac{\Gamma_{t}}{\Gamma_{\mathrm{eq}}}=1-e^{-\frac{D K t}{\delta \Gamma_{\text {lim }}}}
$$

$D$ is a combination of the diffusion coefficient of analyte in free solution and in the hydrogel. Fitting the observed values of peak angle shift for PAB to immobilised streptavidin to an exponential rise gives values for the exponent of $4.86 \times 10^{-3} \mathrm{~s}^{-1}(\mathrm{r}=0.9903)$ and $1.74 \times 10^{-3} \mathrm{~s}^{-1}(\mathrm{r}=0.9793)$ for the unexposed and exposed regions, respectively. The ratio of these two values was 2.79 , while the ratio of $\Gamma_{\text {lim }}$ in the unexposed and exposed regions was 3.88, which implies that one of the other parameters in the expression for the exponential term also differed between the exposed and unexposed regions. Since the dissociation constant $K$ is not expected to be dependent on the (low) density of the binding sites and $\delta$ is dependent only on the dimensions of the flow cell and flow rate, which were the same for all measurements, the only remaining parameter that could vary is the diffusion coefficient $D$. The ratio of diffusion coefficients for PAB in the exposed and unexposed areas is 0.719 . This means that the exposed areas are less porous, even though more streptavidin has been removed.

Fitting of the IgG binding to immobilised PAB to an exponential rise gave a value of the exponent of $9.05 \times 10^{-4} \mathrm{~s}^{-1}(\mathrm{r}=0.9991)$ for the exposed regions and $6.83 \times 10^{-4} \mathrm{~s}^{-1}(\mathrm{r}=0.9990)$ for the unexposed regions. This gives a ratio of the diffusion coefficients in the exposed and unexposed areas of 0.34 , which is consistent with the larger size of IgG compared to PAB if the exposed areas are less porous than the unexposed areas.

\subsubsection{Temperature Compensation during Analyte Unbinding}

To show that the effect of temperature changes could be significantly suppressed while performing analyte binding, a chitosan sensor was prepared and maintained at $20^{\circ} \mathrm{C}$ while PAB was pumped through the flow cell. After a HEPES wash, IgG was allowed to bind to the immobilised PAB, after which a further brief HEPES wash was performed. The temperature of the flow cell was then raised successively to $22.5^{\circ} \mathrm{C}$ and $25.0^{\circ} \mathrm{C}$ and then returned to $20^{\circ} \mathrm{C}$ while HEPES was being pumped through the flow cell. Figure 9 shows the resulting sensorgrams, where (a) is a plot of the absolute resonance angle shifts averaged over three strips of the unexposed (sensing) and exposed (reference) waveguide and (b) is the average difference between the unexposed and exposed areas. Changes in the absolute response are clearly visible as the temperature was changed, but have been significantly reduced in the differential plot. Fitting the data for the HEPES wash with temperature changes to an exponential decay gives an RMS residual error of $0.001382^{\circ}$ for the differential plot and $0.0126^{\circ}$ for the unexposed (sensing) regions. This is a factor of 9 reduction in the effect of temperature. This reduction in sensitivity to temperature, however, does come with a reduction in sensitivity to about half that of the absolute sensitivity of the unexposed regions. 
Time (s)

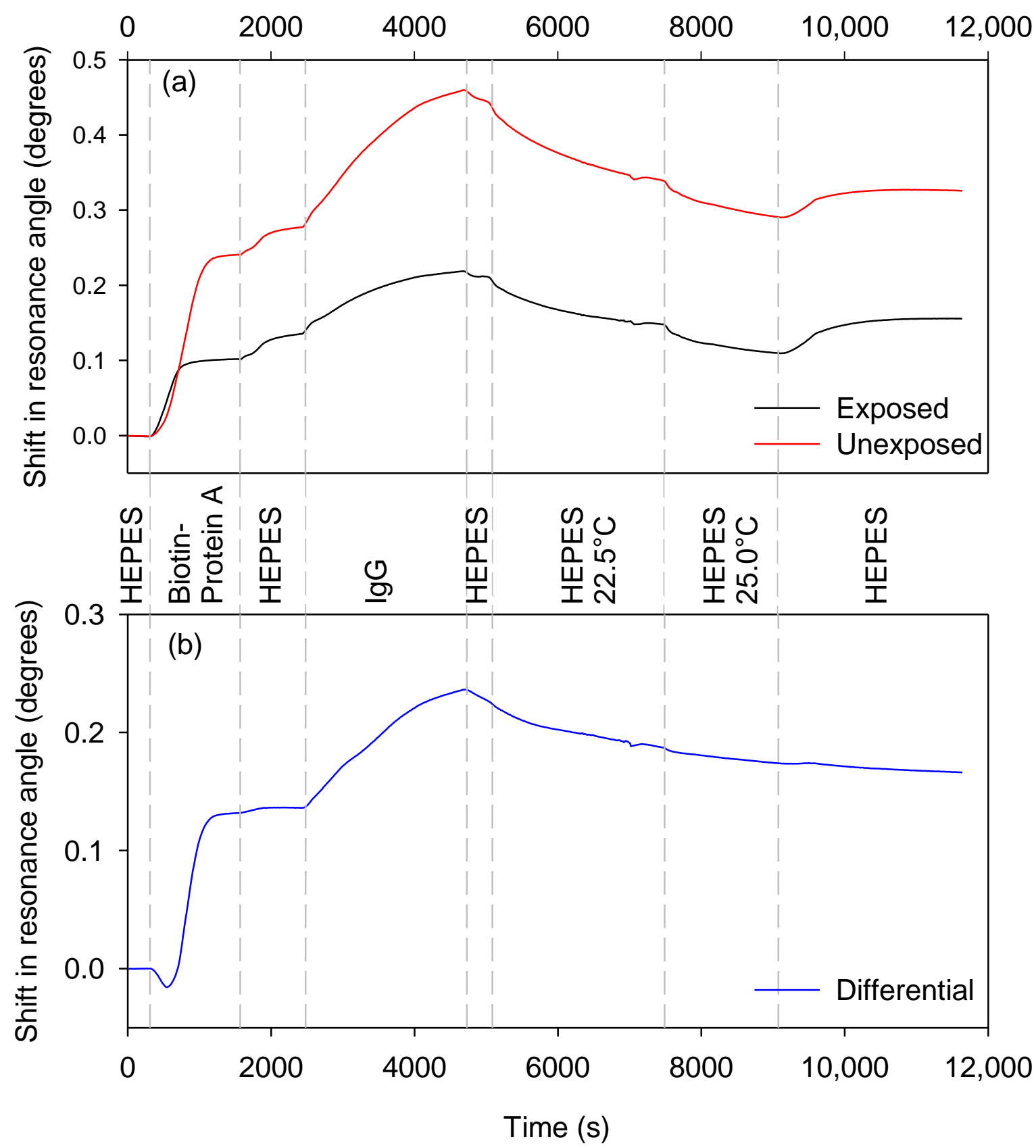

Figure 9. (a) Absolute and (b) differential shifts in the resonance angles of the self-referenced diffraction-based LW to protein A-biotin (PAB) (labelled as biotin-Protein A) and IgG binding and temperature changes during HEPES washing.

Table 2 gives a comparison between our previous work on metal-clad leaky waveguides (MCLWs) comprising stacked agarose waveguide layers and this work on diffraction-based LWs comprising chitosan waveguides in a side-by-side self-referencing configuration. In all cases the present work provides better cancellation of common-mode effects, and provides considerably better differential sensitivity to analyte binding. This is because of the significant overlap between the two modes in the stacked configuration, which does not occur with the side-by-side configuration. The reduction in differential sensitivity in this work is a result of the incomplete removal of streptavidin in the exposed regions and its partial removal in the unexposed regions. 
Table 2. Comparison of our previous work using stacked metal-clad leaky waveguide (MCLW) layers and this work using side-by-side sensing and reference strips.

\begin{tabular}{|c|c|c|c|c|}
\hline \multirow{2}{*}{ Device } & \multirow{2}{*}{$\begin{array}{l}\text { Compensation for } \\
\text { Change in } \\
\text { Temperature }\end{array}$} & \multicolumn{2}{|c|}{$\begin{array}{l}\text { Compensation for Changes in } \\
\text { Sample Composition }\end{array}$} & \multirow{2}{*}{$\begin{array}{l}\text { Reduction in } \\
\text { Sensitivity to } \\
\text { Analyte Binding }\end{array}$} \\
\hline & & $\begin{array}{l}\text { Non-Adsorbing } \\
\text { Interferents }\end{array}$ & $\begin{array}{l}\text { Adsorbing } \\
\text { Interferents }\end{array}$ & \\
\hline Stacked MCLW [34] & $94 \%$ & $97 \%$ & Not tested & $78 \%$ \\
\hline This work & $98 \%$ & $99 \%$ & $97 \%$ & $49 \%$ \\
\hline
\end{tabular}

\section{Conclusions}

Referencing, where a plurality of independent measurements is performed to separate common-mode effects (i.e., changes in temperature, wavelength, and sample composition) from analyte binding, is of paramount importance for practical applications of label-free (optical) biosensors. In this paper, a novel combination of a self-referenced diffraction-based optical leaky waveguide (LW) biosensor with an array of interspersed sensor and reference strips and a pitch of $\sim 1 \mathrm{~mm}$ was fabricated using a photocleavable biotin linker immobilised in chitosan films. The absolute refractive index sensitivity and figure of merit of the LW sensor were $121.09 \pm 4.30^{\circ} \mathrm{RIU}^{-1}$ and $630.68 \pm 22.40 \mathrm{RIU}^{-1}$, respectively. We demonstrated that differential measurements between the sensor and reference regions reduced the effect of changes in temperature over $5{ }^{\circ} \mathrm{C}$ and sample composition because of non-adsorbing and adsorbing species by $\sim 98 \%, \sim 99 \%$, and $\sim 97 \%$, respectively compared to the absolute measurements. The differential sensitivity to analyte binding was reduced to $49 \%$ of the absolute sensitivity, but this decrease in sensitivity was far outweighed by the benefits of compensation for changes in temperature and sample composition. LWs are largely non-dispersive, and hence changes in wavelength have an insignificant effect on their output. Future work will focus on investigating the use of synthetic hydrogels, which offer lower scattering, better control over the density of functional groups, higher batch-to-batch reproducibility, and possibilities for covalent attachment to substrates, for making such self-referenced LW biosensors.

Supplementary Materials: The following are available online at http:/www.mdpi.com/2079-6374/10/10/134/s1, Figure S1: Sensorgram of a chitosan LW irrigated with glycerol solutions prepared in $100 \mathrm{mM}$ HEPES, pH 7.4 at different times where the inset plots shift in resonance angle versus the refractive index of glycerol solutions, Figure S2: Shift in resonance angle of the LW for glycerol and PEG (35, 100 and $300 \mathrm{kD})$ solutions of $10 \mathrm{mg} / \mathrm{mL}$ concentration prepared in $100 \mathrm{mM}$ HEPES, $\mathrm{pH}$ 7.4, Table S1: RI of glycerol solutions of different concentrations measured using RFM900-T refractometer (Bellingham and Stanley, Kent, UK) with an accuracy of $\pm 1 \times 10^{-5}$.

Author Contributions: Conceptualisation, R.G. and N.J.G.; methodology, R.G.; software, N.J.G.; validation, A.K.P., N.J.G., H.J.D. and R.G.; formal analysis, AK.P., N.J.G., H.J.D. and R.G.; investigation, A.K.P. and H.J.D.; resources, R.G.; data curation, A.K.P. and H.J.D.; writing-original draft preparation, A.K.P., N.J.G. and R.G.; writing-review and editing, R.G. and N.J.G.; visualisation, A.K.P., N.J.G. and R.G.; supervision, R.G.; project administration, R.G.; funding acquisition, R.G. Authorship must be limited to those who have contributed substantially to the work reported. All authors have read and agreed to the published version of the manuscript.

Funding: R.G. would like to acknowledge the funding support from the Engineering and Physical Sciences Research Council (Grants EP/N02074X/1 and EP/N02074X/2).

Conflicts of Interest: The authors declare no conflict of interest.

\section{References}

1. Zourob, M.; Goddard, N.J. Metal clad leaky waveguides for chemical and biosensing applications. Biosens. Bioelectron. 2005, 20, 1718. [CrossRef] [PubMed]

2. Osterfeld, M.; Franke, H.; Feger, C. Optical gas-detection using metal-film enhanced leaky mode spectroscopy. Appl. Phys. Lett. 1993, 62, 2310. [CrossRef]

3. Berz, F. On a quarter wave light condenser. Br. J. Appl. Phys. 1965, 16, 1733. [CrossRef]

4. Podgorsek, R.P.; Franke, H. Selective optical detection of aromatic vapors. Appl. Opt. 2002, 41, 601. [CrossRef] 
5. Zhang, Q.W.; Wang, Y.; Mateescu, A.; Sergelen, K.; Kibrom, A.; Jonas, U.; Wei, T.X.; Dostalek, J. Biosensor based on hydrogel optical waveguide spectroscopy for the detection of 17 beta-estradiol. Talanta 2013, 104, 149. [CrossRef]

6. Zourob, M.; Simonian, A.; Wild, J.; Mohr, S.; Fan, X.D.; Abdulhalim, I.; Goddard, N.J. Optical leaky waveguide biosensors for the detection of organophosphorus pesticides. Analyst 2007, 132, 114. [CrossRef]

7. Zourob, M.; Mohr, S.; Brown, B.J.T.; Fielden, P.R.; McDonnell, M.B.; Goddard, N.J. An integrated optical leaky waveguide sensor with electrically induced concentration system for the detection of bacteria. Lab Chip 2005, 5, 1360. [CrossRef]

8. Alamrani, N.A.; Greenway, G.M.; Pamme, N.; Goddard, N.J.; Gupta, R. A feasibility study of a leaky waveguide aptasensor for thrombin. Analyst 2019, 144, 6048. [CrossRef]

9. Im, W.J.; Kim, B.B.; Byun, J.Y.; Kim, H.M.; Kim, M.G.; Shin, Y.B. Immunosensing using a metal clad leaky waveguide biosensor for clinical diagnosis. Sens. Actuators B-Chem. 2012, 173, 288. [CrossRef]

10. Kim, B.B.; Im, W.J.; Byun, J.Y.; Kim, H.M.; Kim, M.G.; Shin, Y.B. Label-free CRP detection using optical biosensor with one-step immobilization of antibody on nitrocellulose membrane. Sens. Actuators B-Chem. 2014, 190, 243. [CrossRef]

11. Der, A.; Valkai, S.; Mathesz, A.; Ando, I.; Wolff, E.K.; Ormos, P. Protein-based all-optical sensor device. Sens. Actuators B-Chem. 2010, 151, 26. [CrossRef]

12. Goddard, N.J.; Gupta, R. A novel manifestation at optical leaky waveguide modes for sensing applications. Sens. Actuators B-Chem. 2020, 309, 127776. [CrossRef]

13. Gupta, R.; Goddard, N.J. Leaky waveguides (LWs) for chemical and biological sensing-A review and future perspective. Sens. Actuators B Chem. 2020, 322, 128628-128642. [CrossRef]

14. Goddard, N.J.; Gupta, R. Speed and sensitivity-Integration of electrokinetic preconcentration with a leaky waveguide biosensor. Sens. Actuators B Chem. 2019, 301, 127063. [CrossRef]

15. Thormahlen, I.; Straub, J.; Grigull, U. Refractive-index of water and its dependence on wavelength, temperature, and density. J. Phys. Chem. Ref. Data 1985, 14, 933. [CrossRef]

16. Abbate, G.; Bernini, U.; Ragozzino, E.; Somma, F. The temperature dependence of the refractive index of water. J. Phys. D Appl. Phys. 1978, 11, 1167. [CrossRef]

17. Manninen, P.; Orrevetelainen, P. On spectral and thermal behaviors of AlGaInP light-emitting diodes under pulse-width modulation. Appl. Phys. Lett. 2007, 91, 181121. [CrossRef]

18. Yakes, B.J.; Buijs, J.; Elliott, C.T.; Campbell, K. Surface plasmon resonance biosensing: Approaches for screening and characterising antibodies for food diagnostics. Talanta 2016, 156, 55. [CrossRef]

19. Lu, H.B.; Homola, J.; Campbell, C.T.; Nenninger, G.G.; Yee, S.S.; Ratner, B.D. Protein contact printing for a surface plasmon resonance biosensor with on-chip referencing. Sens. Actuators B-Chem. 2001, 74, 91. [CrossRef]

20. Yu, F.; Knoll, W. Immunosensor with self-referencing based on surface plasmon diffraction. Anal. Chem. 2004, 76, 1971. [CrossRef]

21. Nizamov, S.; Scherbahn, V.; Mirsky, V.M. Self-referencing SPR-sensor based on integral measurements of light intensity reflected by arbitrarily distributed sensing and referencing spots. Sens. Actuators B-Chem. 2015, 207, 740. [CrossRef]

22. Zou, Q.J.; Menegazzo, N.; Booksh, K.S. Development and investigation of a dual-pad in-channel referencing surface plasmon resonance sensor. Anal. Chem. 2012, 84, 7891. [CrossRef] [PubMed]

23. Wegner, S.V.; Senturk, O.I.; Spatz, J.P. Photocleavable linker for the patterning of bioactive molecules. Sci. Rep. 2015, 5. [CrossRef]

24. Pal, A.K.; Labella, E.; Goddard, N.J.; Gupta, R. Photofunctionalizable hydrogel for fabricating volume optical diffractive sensors. Macromol. Chem. Phys. 2019, 220, 1900228. [CrossRef]

25. Gupta, R.; Alamrani, N.; Greenway, G.M.; Pamme, N.; Goddard, N.J. A method for determining average iron content of ferritin by measuring its optical dispersion. Anal. Chem. 2019, 91, 7366. [CrossRef]

26. Löfås, S.; Johnsson, B. A novel hydrogel matrix on gold surfaces in surface plasmon resonance sensors for fast and efficient covalent immobilization of ligands. J. Chem. Soc. Chem. Commun. 1990, 1526-1528. [CrossRef]

27. Cush, R.; Cronin, J.M.; Stewart, W.J.; Maule, C.H.; Molloy, J.; Goddard, N.J. The resonant mirror-A novel optical biosensor for direct sensing of biomolecular interactions. 1. Principle of operation and associated instrumentation. Biosens. Bioelectron. 1993, 8, 347. [CrossRef] 
28. Gupta, R.; Goddard, N.J.; Dixon, H.; Toole, N. 3D printed instrumentation for point-of-use leaky waveguide (LW) biochemical sensor. IEEE Trans. Instrum. Meas. 2020, 69, 6390. [CrossRef]

29. Zhao, H.Y.; Brown, P.H.; Schuckt, P. On the distribution of protein refractive index increments. Biophys. J. 2011, 100, 2309. [CrossRef]

30. Jachimska, B.; Pajor, A. Physico-chemical characterization of bovine serum albumin in solution and as deposited on surfaces. Bioelectrochemistry 2012, 87, 138. [CrossRef]

31. Mohammed, M.A.; Syeda, J.T.M.; Wasan, K.M.; Wasan, E.K. An Overview of Chitosan Nanoparticles and Its Application in Non-Parenteral Drug Delivery. Pharmaceutics 2017, 9, 53. [CrossRef] [PubMed]

32. Wang, Y.J.; Wang, X.H.; Luo, G.S.; Dai, Y.Y. Adsorption of bovin serum albumin (BSA) onto the magnetic chitosan nanoparticles prepared by a microemulsion system. Bioresour. Technol. 2008, 99, 3881-3884. [CrossRef] [PubMed]

33. Eddowes, M.J. Direct immunochemical sensing-Basic chemical principles and fundamental limitations. Biosensors 1987, 3, 1-15. [CrossRef]

34. Gupta, R.; Goddard, N.J. A novel optical biosensor with internal referencing. In Proceedings of the 17th International Conference on Miniaturized Systems for Chemistry and Life Sciences, Freiburg, Germany, 27-31 October 2013; p. 1490.

(C) 2020 by the authors. Licensee MDPI, Basel, Switzerland. This article is an open access article distributed under the terms and conditions of the Creative Commons Attribution (CC BY) license (http://creativecommons.org/licenses/by/4.0/). 